\title{
Locomotion Patterns based on a CPG Model of Snake Robot
}

\author{
Wei-Jian HU1', Lan-Ying ZHAO',a, Jun-Li YAN', Jin-Xiao LI $^{2}$, Zhe-Long WANG ${ }^{2}$ \\ ${ }^{1}$ National Earthquake Response Support Service, Beijing, China \\ ${ }^{2}$ School of Control Science and Engineering, Dalian University of Technology, Dalian, China \\ alyzhao@cugb.edu.cn
}

\begin{abstract}
Keywords: Locomotion Patterns, CPG Network Model, Laboratory Simulation, Snake Robot.
\end{abstract}
\begin{abstract}
The large number and scales of earthquake disasters have occurred around the world in the past decade. In order to mitigate damages from earthquake disasters, the intelligent rescue systems with high information technology and robot technology have been expected. With the rapid development of rescue robot, snake robots have great potential in the search and rescue operation of the earthquake disasters due to their slim and flexible bodies. In this paper, a novel control method for a snake robot is proposed. Our method can generate different types of locomotion pattern in the complex terrain, and it may employ a central pattern generator (CPG) model based on Hopf oscillators in order to acquire desired locomotion patterns. The simulation results of the proposed method show that the proposed method is feasible.
\end{abstract}

\section{Introduction}

Inspired by locomotion control of biological snakes, snake robots have broad application prospect in complicated environment. In recent years, the frequent occurrence of earthquake disasters causes incalculable human damages and economic losses to the society. Especially earthquakes cause the large area housing collapse, ground collapse and so on, this has brought great difficulty to people. Because vertebrate rhythmic movement is controlled by central pattern generator (CPG), it has been proven that the mechanism of snake is more flexible and adaptable in difficult environments than the ordinary locomotion mechanisms with wheels or legs. The control strategy based on CPG has favourable auto stability and adaptability, so it is applied to motion control of robot [1-4].

In the literature a variety of CPG models based on the oscillator were used in snake robots. In 2005, Ijspeert [5-6] et al. employed distributed nonlinear oscillator to construct CPG gait controller, and realized successfully two movements of the snake robot in land and water. In 2007, Inoue [7] designed a CPG network with multiple topologies based on Matsuoka model, and used genetic algorithm to adjust CPG parameters. On the basis of the Matsuoka oscillator model, Ryu [8] designed a link network with adaptive frequency characteristics in 2009. Wu [9-10] presented a CPG network model with feedback connection based on cyclic inhibition model in 2010, and discussed the relationship between model parameters and output signal. In the published literature much works about snake robot had been analysed [11-12], but there are still a lot of challenges about the modelling and control of the snake robot due to the multiple degrees of freedom and high controlled difficulty of snake robot.

Our goal is to realize effective locomotion of snake robot in complicated environment by designing a CPG model based on a Hopf oscillator. According to the structure and movement characteristics of the biological snake, this paper presents the CPG model of snake robot that use a coupled Hopf oscillator with feature of nonlinear limit cycle. In the paper we study the relationship between the main parameters of the oscillator and the speed of the robot. Simulation and experiments were carried out to determine the effectiveness of the proposed control strategy.

\section{Design and Control of Snake Robot}

This section gives an overview related to design and control of our snake robot. The design of snake robot connects always in series joint units, and a central pattern generator (CPG) model based method 
of motion control is proposed.

In order to realize the desired motion of the snake robot in three-dimensional space, the designed joint module should have at least two degrees of freedom. We can usually use the characteristics of the universal joint structure to achieve it. According to the principle of reconfigurable, we design a snake robot prototype with single joint and two degrees of freedom.

The snake robot consists of eleven links which are connected through active joints in the design. The joint module of snake robot, as shown in Fig. 1, mainly contains two small DC motors, two motor housings, two bevel gear set, passive wheels and an active ring. Two motors drive directly a revolute joint which includes two degrees of freedom.

One is used to control the joint angle between two links on the horizontal plane, and the other is used to control the joint angle between two links on the vertical plane.

The whole snake robot prototype, as shown in Fig. 2, is structured in series by eleven identical cylinder connecting rod modules. Active joint module is structured by cylinder connecting rod through swiveling ring. Our design uses six passive wheels that fixed on the cylinder body in the circular uniform distribution. After the structure is improved, our snake robot will be more flexible and adaptable.

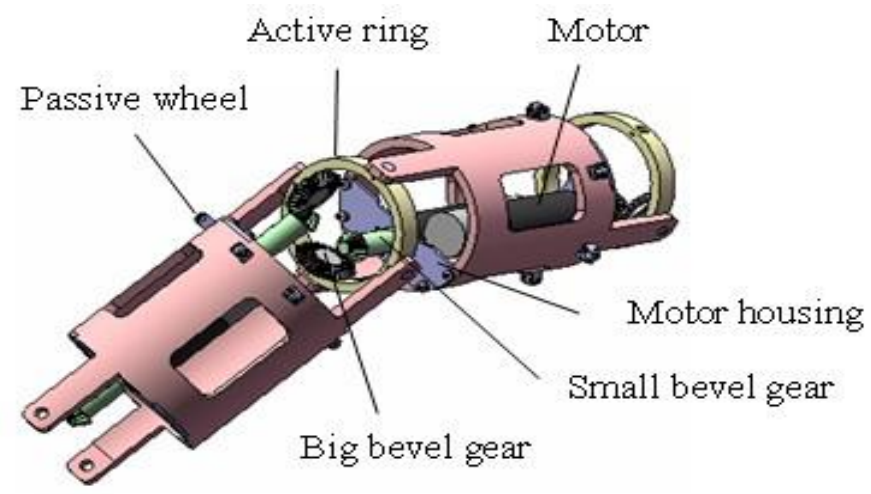

Fig. 1. The structure of a joint of our snake robot

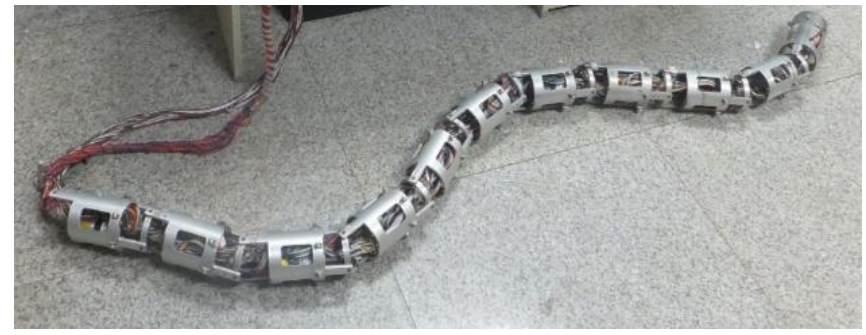

Fig. 2.The snake robot prototype

\section{Equations CPG Network Model based on Oscillators}

\section{Hopf Oscillator}

The dynamic characteristics of the Hopf oscillator can be expressed as:

$$
\dot{x}=f_{H}(x)=\left[\begin{array}{c}
-\lambda\left(\frac{u^{2}+v^{2}}{r^{2}}-\sigma\right) u+\omega(t) v \\
-\omega(t) u+-\lambda\left(\frac{u^{2}+v^{2}}{r^{2}}-\sigma\right) v
\end{array}\right]
$$

where $x=[u, v]^{T}, \dot{x}=f_{H}(x-o(t)), o(t)$ is defined as oscillation center of state $x$. The frequency of limit cycle of oscillator is determined by $\omega(t), r$ is the radius of the limit cycle on the plane, $\lambda$ is defined as the attractive rate which convergence to the limit cycle, $\sigma$ is the Hopf bifurcation 
parameter. Nonlinear limit cycles are suitable for constructing dynamic models.

\section{CPG Network Model}

Aiming at the snake robot with single joint and two degrees of freedom, we present a CPG network that can achieve serpentine locomotion and sidewinding locomotion. The CPG network is shown in Figure. 3.

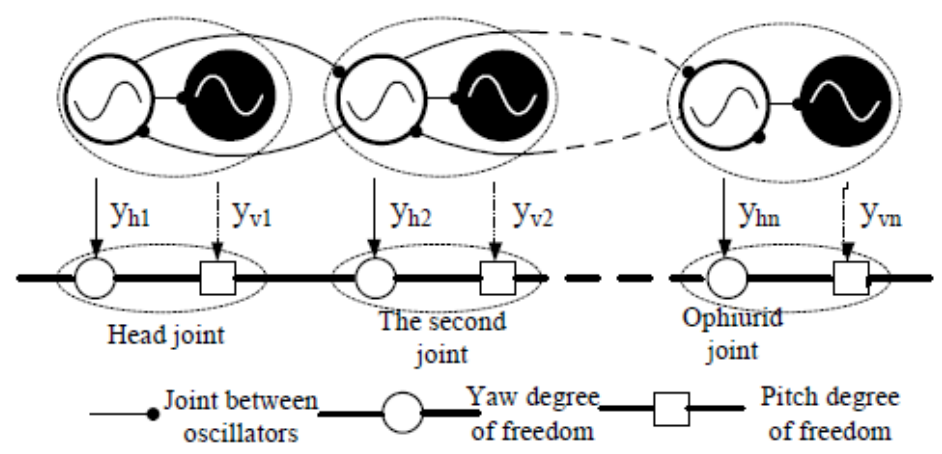

Fig. 3. A CPG network consisting of 2n Hopf oscillators

The oscillators in the graph can be divided into two types that white circle represents horizontal Hopf oscillator and black circle represents vertical Hopf oscillator, and each oscillator corresponds to a degree of freedom. While horizontal oscillator group produce synchronous oscillation signal,all the vertical oscillators are simultaneously implemented since they always keep a fixed phase difference with the horizontal oscillator. Such characteristic is advantageous to the realization of the sidewinding gait, that is to say, there is a fixed phase difference between swing angle function on the horizontal plane and swing angle function on the vertical plane corresponding to the same joint.

In the CPG networks of the snake robot in Fig. 3, the Hopf oscillator model of controlling the $i$ th joint is as follows:

$$
\begin{aligned}
& \dot{x}_{i}=f_{H}\left(x_{i}-o_{i}\right)-\alpha \sum_{j} w_{i j}\left(x_{i}-o_{i}-\frac{r_{x i}}{r_{x j}} R\left(\phi_{i j}\right) x_{j}\right) \\
& \dot{y}_{i}=f_{H}\left(y_{i}\right)-\beta\left(y_{i}-\frac{r_{x i}}{r_{x j}} R\left(\phi_{y x}\right) x_{j}\right) \\
& \theta_{h, i}=x_{i}^{(1)} \\
& \theta_{v, i}=y_{i}^{(1)}
\end{aligned}
$$

where

$$
R\left(\phi_{i j}\right)=\left[\begin{array}{cc}
\cos \phi_{i j} & -\sin \phi_{i j} \\
\sin \phi_{i j} & \cos \phi_{i j}
\end{array}\right]
$$

the state variables of the horizontal oscillator and vertical oscillator respectively are $x_{i}=\left[u_{x i}, v_{x i}\right]^{T}$, $y_{i}=\left[u_{y i}, v_{y i}\right]^{T}, i=1,2, \cdots, n, o_{i}$ is the symmetric center of the $i$ th horizontal oscillator, $\alpha, \beta$ are respectively coupling gain of the horizontal oscillator and vertical oscillator. $r_{x i}$ and $r_{y i}$ are respectively amplitude of the $i$ th horizontal oscillator and vertical oscillator, $\phi_{i j}$ is the phase difference from the $j$ th horizontal oscillator to the $i$ th horizontal oscillator, $\phi_{y x}$ is the phase difference from the $i$ th horizontal oscillator to the $i$ th vertical oscillator.

If the balance of the weight coupling matrix can cause the Hopf limit cycle system to exponential convergence to steady state, the CPG network composed of the oscillators can generate the rhythm 
signal. For concreteness when the oscillator network employs the mode of diffusion bonding, the weighted connection matrix is balanced, that is to say,

$$
\sum_{i \neq j} w_{i j}=\sum_{i \neq j} w_{j i}
$$

Then we can realize synchronization of the whole oscillator system when the global coupling gain value is increased to a certain extent. In order to realize synchronous oscillator, the connection weights between the oscillators can be selected as:

$$
w_{i j}=\left\{\begin{array}{l}
a, j=i-1, \text { and } i=2,3, \cdots, n \\
b, j=i+1, \text { and } i=1,2, \cdots, n \\
0, \text { else }
\end{array}\right.
$$

where $a, b>0$, the connection weights of the oscillators from head to tail are $a$, the phase difference between adjacent oscillators is $\phi_{i, i-1}$. The connection weights of the neighbouring oscillators from tail to head are $b$, the phase difference between adjacent horizontal oscillators is $\phi_{i-1, i}$. When the oscillators are synchronous, we have $\phi_{i, i-1}=-\phi_{i-1, i}$. Generally speaking joint configuration of the whole snake robot forms a $\mathrm{S}$ waveform, so the phase difference of the joint angle change of the design satisfies $\phi_{i, i-1}=2 \pi / n$.

\section{Experience}

In this section, in order to confirm the validity of the control strategy based on the CPG, we realize the simulation of serpentine locomotion and sidewinding locomotion of snake robot in the horizontal plane, as shown in Fig. 4. In the serpentine locomotion, the horizontal oscillator of CPG networks are dependent on the parameters $r_{x i}=\pi / 9, \omega_{x i}=\pi / 3, \phi_{i, j}=\pi / 5$. In the sidewinding locomotion, the horizontal oscillator are dependent on the parameters $r_{x i}=\pi / 9, \omega_{x i}=\pi / 4, \phi_{i, j}=\pi / 5$, and the vertical oscillators are dependent on the parameters $r_{y i}=\pi / 36, \omega_{y i}=\pi / 4, \phi_{y, x}=\pi / 2$.

From the Fig. 5 and Fig. 6, it can be seen that the serpentine locomotion and sidewinding locomotion in the laboratory are realized. Then we find some differences between the experimental result and simulation result, since the structure and circumstance of the snake robot cannot be completely fit to simulation setting. Through repeated experiments, we find the deflection of the forward direction of the robot. And when the robot moves in sidewinding locomotion, the fluctuation law of the joints of vertical degrees of freedom is not obvious.

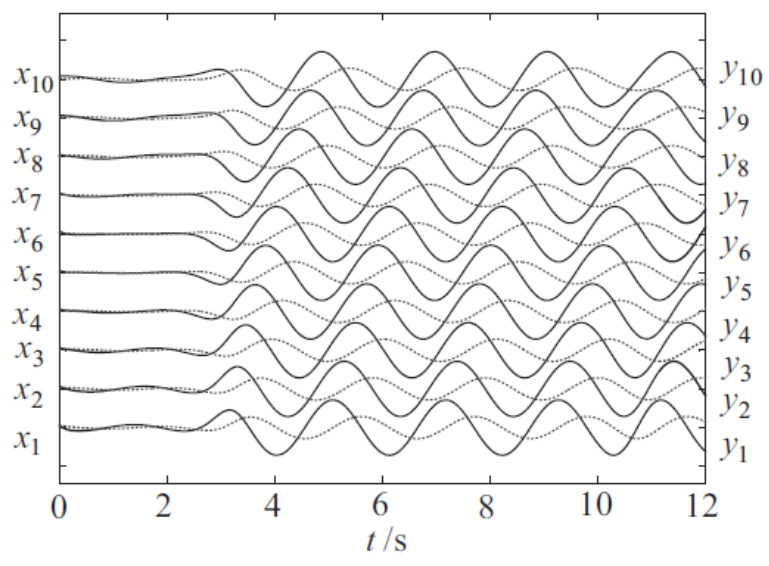

Fig. 4. Output signals of the 20 Hopf oscillators in CPG network 


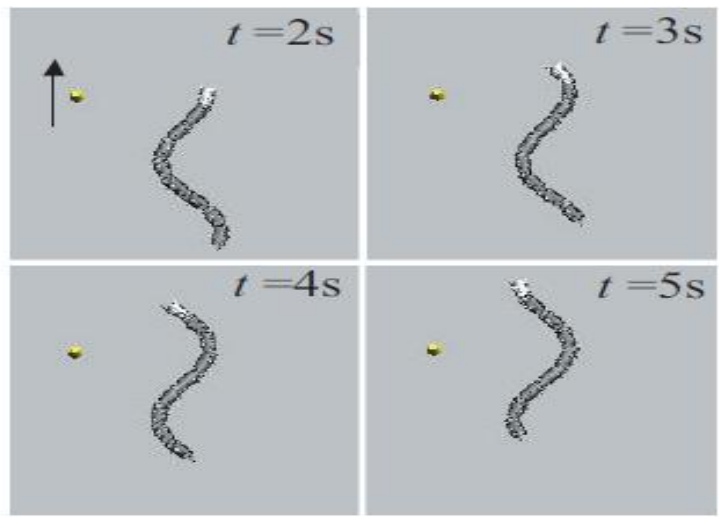

Fig. 5. The experimental result of serpentine locomotion

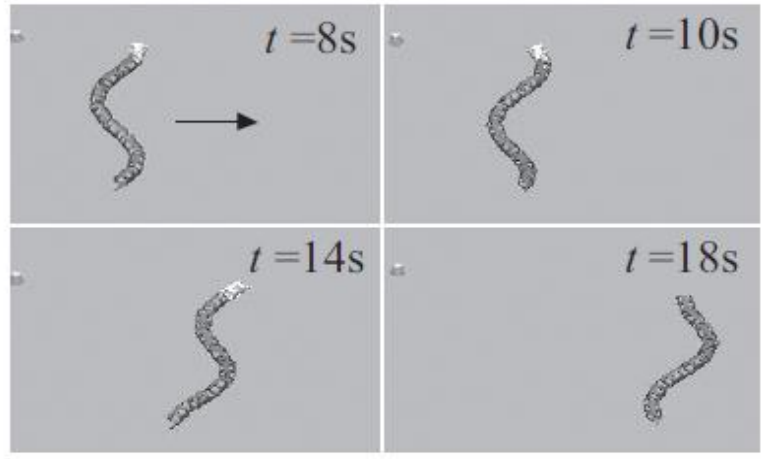

Fig. 6. The experimental result of sidewinding locomotion

\section{Conclusions}

In the paper we design a snake robot based on the central pattern generator (CPG) with joint of two degrees of freedom and realize its serpentine locomotion and sidewinding locomotion which is controlled easily. Experimental results of the snake robot show that the serpentine locomotion is reasonable and the snake robot moves forward smoothly. With the CPG network we can utilize few effective control parameters to affect directly multiple motion performance of the robot, and improve the adaptability of the robot.

However, there are still a lot of works that need to solve. Future applications of snake robots will generally require more research on adaptive behavior during motion in unknown and cluttered environments.

\section{Acknowledgment}

This program is supported by the National Key Technology Research and Development Program of China under Grant Number 2013BAK03B01.

\section{References}

1. Taga G, Yamaguchi Y, Shimizu H., J. Biol. Cybern. 65, 3 (1991).

2. Miyakoshi S, Taga G, Kuniyoshi Y, et al., IEEE/RSJ IROS, (1998).

3. Kimura H, Fukuoka Y, Nakamura H., ISRR (2000).

4. Morel Y, Porez M, Leonessa A, et al., IEEE, CDC-ECC (2011).

5. Crespi A, Badertscher A, Guignard A, et al., J. Robot. Auton. Syst., 50, 4, (2005).

6. Crespi A, Ijspeert A J. AmphiBot II., 9th CLAWAR (2006). 
7. Inoue K, Sumi T, Ma S G., IEEE /RSJ IROS (2007).

8. Ryu J K, Chong N Y, You B J, et al., J. Intel. Serv. Robot. 3, 1(2009).

9. Wu X D, Ma S G., J. Auton. Robot. 28, 3(2010).

10. Wu X D, Ma S G. J. Mechatronics, 20, 2 (2010).

11. Matsuoka K., J. Biol. Cybern. 104, 4 (2011).

12. Gao Q, Wang Z, Shang H, et al., Advances in Intelligent Systems and Computing (2013). 